\title{
Active Microstructured Optical Arrays of Grazing Incidence Reflectors
}

\author{
Richard Willingale, ${ }^{1}$ Charlotte Feldman, ${ }^{1}$ Alan Michette, ${ }^{2}$ Tim Button, ${ }^{3}$ Camelia Dunare, \\ Melvyn Folkard, ${ }^{5}$ David Hart, ${ }^{2}$ Chris McFaul, ${ }^{2}$ Graeme R. Morrison, ${ }^{2}$ William Parkes, ${ }^{4}$ \\ Sławka Pfauntsch, ${ }^{2}$ A. Keith Powell, ${ }^{2}$ Daniel Rodriguez-Sanmartin, ${ }^{3}$ Shahin Sahraei, ${ }^{2}$ \\ Matthew T. Shand, ${ }^{2}$ Tom Stevenson, ${ }^{4}$ Boris Vojnovic, ${ }^{5,6}$ and Dou Zhang ${ }^{3}$ \\ ${ }^{1}$ Department of Physics and Astronomy, University of Leicester, Leicester LE1 7RH, UK \\ ${ }^{2}$ Department of Physics, Kings College London, Strand, London WC2R 2LS, UK \\ ${ }^{3}$ Department of Metallurgy and Materials, University of Birmingham, Birmingham B15 2TT, UK \\ ${ }^{4}$ Scottish Microelectronics Centre, University of Edinburgh, The Kings Buildings, West Mains Road, Edinburgh EH9 3JF, UK \\ ${ }^{5}$ Gray Cancer Institute, Old Road Campus, Roosevelt Drive, Headington, Oxford, Oxfordshire OX3 7DQ, UK \\ ${ }^{6}$ Randall Division of Cell and Molecular Biophysics, Randall Institute, New Hunt's House, King's College London, \\ Guy's Campus, London, SE1 1UL, UK
}

Correspondence should be addressed to Richard Willingale, rw@star.le.ac.uk

Received 22 September 2009; Accepted 16 June 2010

Academic Editor: Ali Khounsary

Copyright (C) 2010 Richard Willingale et al. This is an open access article distributed under the Creative Commons Attribution License, which permits unrestricted use, distribution, and reproduction in any medium, provided the original work is properly cited.

The UK Smart X-Ray Optics (SXO) programme is developing active/adaptive optics for terrestrial applications. One of the technologies proposed is microstructured optical arrays (MOAs), which focus X-rays using grazing incidence reflection through consecutive aligned arrays of microscopic channels. Although such arrays are similar in concept to polycapillary and microchannel plate optics, they can be bent and adjusted using piezoelectric actuators providing control over the focusing and inherent aberrations. Custom configurations can be designed, using ray tracing and finite element analysis, for applications from sub$\mathrm{keV}$ to several-keV X-rays, and the channels of appropriate aspect ratios can be made using deep silicon etching. An exemplar application will be in the microprobing of biological cells and tissue samples using $\mathrm{Ti}_{\alpha}$ radiation ( $4.5 \mathrm{keV}$ ) in studies related to radiation-induced cancers. This paper discusses the optical design, modelling, and manufacture of such optics.

\section{Introduction}

Microoptical arrays (MOAs) is the generic name for optical systems consisting of a very large number of small (microscopic) elements. Such elements can be refractive (lenses), diffractive (gratings), or reflective (mirrors). The idea of MOAs of grazing incidence reflectors was first discussed at a SPIE meeting in 2001 [1] and subsequently at the international X-ray Microscopy conference in Grenoble in 2002 [2]. Some further development has been reported since then $[3,4]$, and the UK Smart X-ray Optics consortium (SXO) has received funds initially for a pilot study (2004) and a full programme of research (starting 2006) under the Council UKs' Basic Technology scheme [5, 6]. Initial work on
$\mathrm{X}$-ray MOAs in the SXO programme was reported at SPIE in 2007 [7] and in 2009 [8-10].

The MOA concept is similar to polycapillary [11] and microchannel plate [12] optics, in which X-rays are guided by multiple grazing incidence reflections along a large number of small channels. The arrays can be manufactured by etching a periodic array of narrow channels in silicon wafers as illustrated in Figure 1. In this sample, the channels are parallel, $\approx 10 \mu \mathrm{m}$ wide, with a pitch of $\approx 20 \mu \mathrm{m}$. The sides of the channels act as mirrors at grazing incidence to provide point-to-point or line-to-line imaging/focusing as shown in Figure 2. If the channels are planar then we can get line-to-line focusing via just 1 reflection or spot-tospot focusing using two successive orthogonal reflections. 


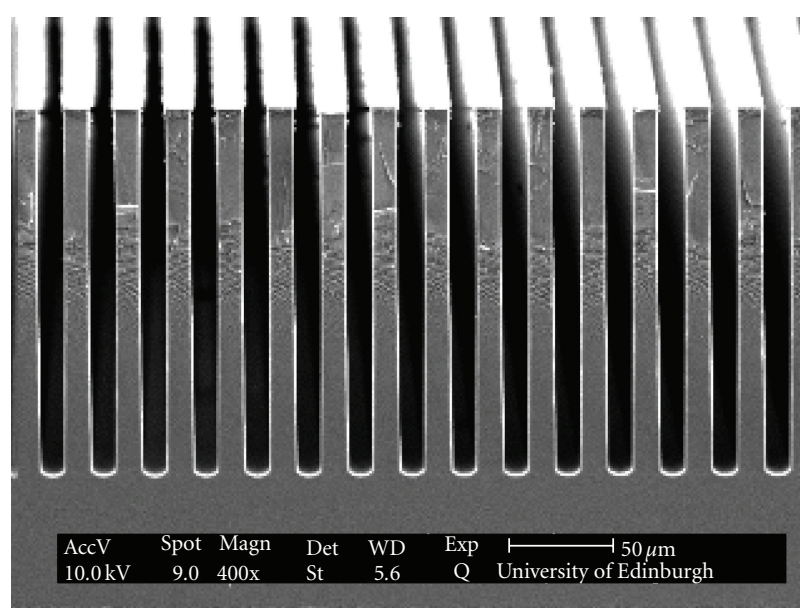

FIGURE 1: MOA channels etched in Si (SMC UoE).

If a more ambitious circular/radial slot geometry is adopted and 2 reflections are employed, then we can get point-topoint imaging as illustrated in Figure 3. The use of two reflections means that the Abbe sine condition can be approximately satisfied, thereby greatly reducing aberrations (in particular coma). A central stop prevents unreflected $\mathrm{X}$-rays from reaching the focus. In practice many more channels would be used than those illustrated. Through actively varying the radius of curvature of one of the reflection arrays by actuating piezoelectric material fixed to the radial spoke the focal length can be changed.

In Section 2, we consider the geometric optics of several possible MOA arrangements suitable for focusing X-rays. The manufacturing of the channels by etching and the actuators attachment are discussed in Section 3 and X-ray scattering from the surface roughness and profile errors on the channel walls are discussed in Section 4. Ray-tracing and finite element analysis of a 1-D MOA structure are presented in Section 5, and finally, Section 6 describes the application of X-ray focusing by MOAs in microprobing of biological cells.

\section{The Geometric Optics of Grazing Incidence Microstructured Optical Arrays}

We will start by considering line-to-line focusing using a single-reflection MOA consisting of planar channels, as shown schematically in Figure 2. We will then discuss tandem systems which use two reflections; firstly in the same plane to provide line-to-line focusing and secondly in perpendicular crossed planes to provide spot-to-spot focusing. Finally, we will look at the generalisation to spot-to-spot focusing using channels with a circular geometry (as shown in Figure 3) which is obvious via symmetry arguments.

2.1. Line-to-Line MOA Focusing. For an unbent component the object and image distances are equal, or more generally, using a bent component such that the channel walls are tilted, we have the geometry shown in Figure 4. The combined reflections from many channels only provide an

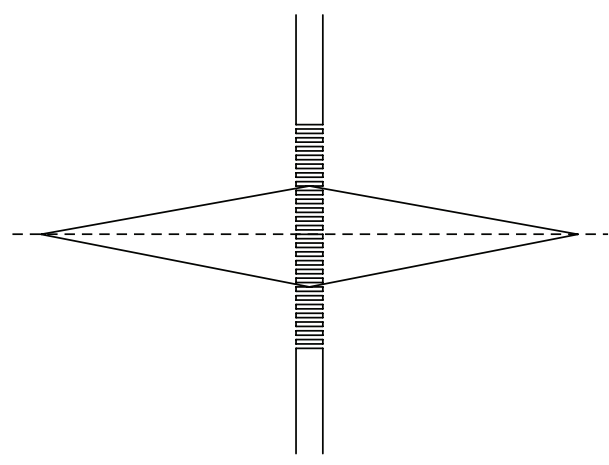

FIGURE 2: Grazing incidence reflections from the slot walls provide line-to-line focusing.

approximate line focus because the reflecting surfaces are assumed to be planar rather than curved. If the length of the channels is $l$ and the grazing angle is $\theta_{g}$, then the focused line width will be $\simeq l \theta_{g}$. The geometry of a single-reflection from a channel wall is shown in Figure 4; the source to MOA distance is $s_{1}$ and the MOA to image distance is $s_{2}$. The channel is tilted by an angle $\varphi$ with respect to the optical axis. If the distance of the channel wall from the axis is $h$, then, using the small angle approximation $\left(h \ll s_{1}, s_{2}\right)$,

$$
\begin{aligned}
\frac{h}{s_{1}} & =\theta_{g}-\varphi, \\
\frac{h}{s_{2}} & =\theta_{g}+\varphi .
\end{aligned}
$$

Eliminating $\varphi$ leads to

$$
\frac{1}{s_{1}}+\frac{1}{s_{2}}=\frac{2 \theta_{g}}{h}=\frac{1}{f}
$$

which is the standard Gaussian imaging equation with a focal length $f=h / 2 \theta_{g}$. Eliminating $\theta_{g}$ between (1) gives

$$
\frac{1}{s_{2}}-\frac{1}{s_{1}}=\frac{2 \varphi}{h}
$$

which gives the MOA radius of curvature, $R=h / \varphi$,

$$
R=\frac{2 s_{1} s_{2}}{s_{1}-s_{2}} .
$$

Note that if $s_{2}=s_{1}$, the radius of curvature is infinite and the MOA is unbent. If the channel width is $w$, then there is a maximum grazing angle of operation, $\theta_{g \max }=2 w / l$, assuming parallel walls (see Figure 5). This, in turn, sets an upper limit to the separation from the optical axis, $h_{\max }=2 s_{1} w / l$ and hence, assuming no channel curvature, defines the effective $f$-ratio of the optic, $2 h_{\max } / f=8 w / l$.

The aperture can be increased by employing a pair of MOAs so that the focusing is accomplished by two reflections. The grazing angles on each component are then reduced by a factor of approximately two for a given value of $h$. The increase in the effective aperture depends on the ratio of the grazing angles in the two-components. Such an arrangement is shown schematically in Figure 6. 


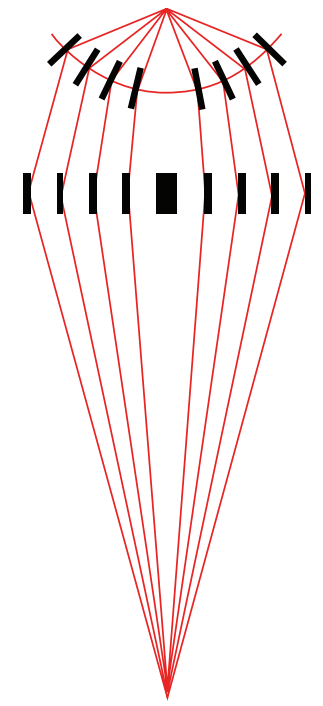

(a)

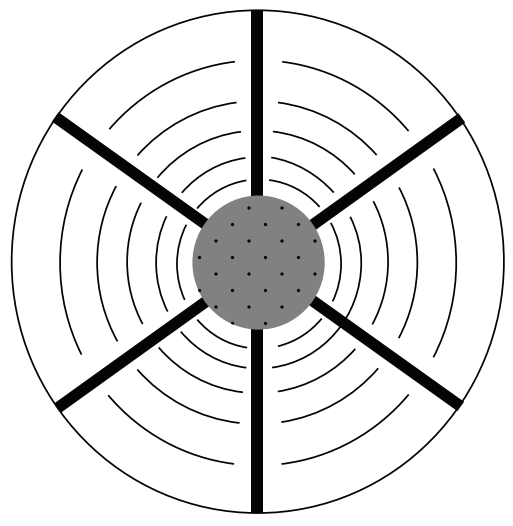

(b)

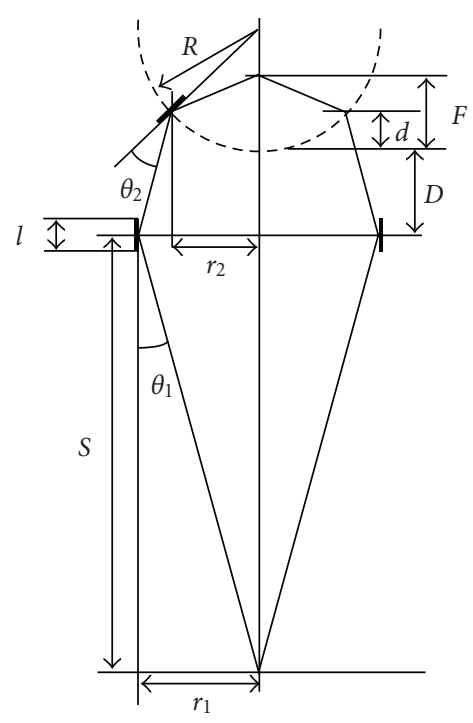

(c)

Figure 3: (a) Schematic of a two-reflection MOA. (b) Possible channel layout of one of the components. (c) Definition of the geometry of a two-reflection MOA.

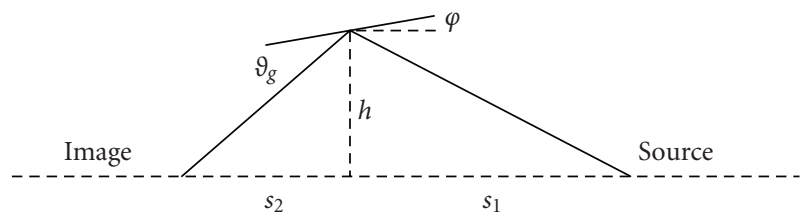

FIGURE 4: Geometry of a single-reflection.

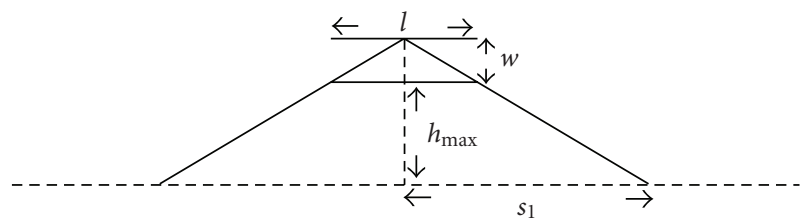

FIGURE 5: The maximum grazing angle for a channel of length $l$ and width $w$.

Three cases of a two component (2-reflection) MOA are considered below:

(i) The grazing angle is the same in each of the components, which are bent in opposite directions (defined to be negative curvature for the first component and positive for the second). This is likely to yield closeto-maximum efficiency for the double-reflections which, for linear arrays, produce a line image. The effective aperture will be double that obtained with a single MOA, $2 h_{\max } / f=16 \mathrm{w} / \mathrm{l}$.

(ii) One component is flat and the other is curved.

(iii) The focal length of the first component is set to the object distance $s_{1}$ and that of the second component to the image distance $s_{2}$.

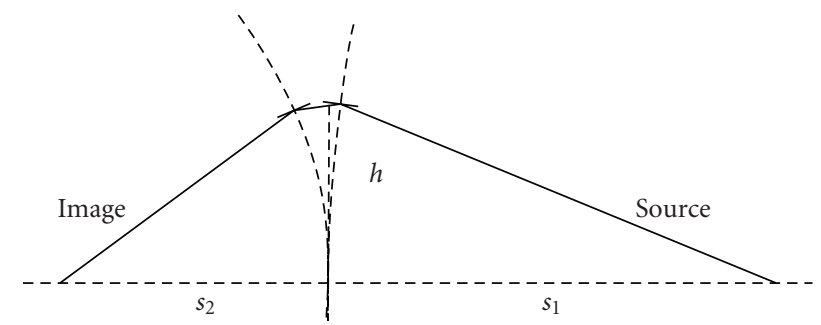

FIGURE 6: In-plane reflection from a two-component MOA.

In the third case, the pair of plates forms a collimatortelescope combination; rays passing from the first to the second component are parallel to the optical axis (planar wave fronts). If the channel sizes and spacings are identical for both components then all rays reflected from the first will intersect with the reflecting surfaces of the second. However, in the first two cases, the rays are converging or diverging and the fraction of rays reflected by the second component will depend on the separation between the plates.

For the reflections from the two components

$$
\frac{h_{1}}{s_{1}}=\theta_{g 1}+\varphi_{1}, \quad \frac{h_{2}}{s_{2}}=\theta_{g 2}+\varphi_{2} .
$$

2.1.1. Equal Grazing Angle Double-Reflection MOAs. Setting the grazing angles to be equal $\left(\theta_{g 1}=\theta_{g 2}=\theta_{g}\right)$, eliminating them from (5), using $\varphi_{1}=-h_{1} / R_{1}$ and $\varphi_{2}=h_{2} / R_{2}$, and assuming that the component separation $D \ll s_{1}, s_{2}$ so that $h_{2} \approx h_{1} \approx h$, then

$$
\frac{1}{R_{1}}+\frac{1}{R_{2}}=\frac{1}{s_{2}}-\frac{1}{s_{1}}
$$


The included angle between the two components is equal to $\Pi-\varphi_{1}-\varphi_{2}$ and to $\Pi-2 \theta_{g}$. It then follows that

$$
\frac{1}{R_{2}}-\frac{3}{R_{1}}=\frac{2}{s_{1}}
$$

and solving (6) and (7) for the radii of curvature $R_{1}$ and $R_{2}$ gives

$$
R_{1}=\frac{4 s_{1} s_{2}}{s_{1}-3 s_{2}}, \quad R_{2}=\frac{4 s_{1} s_{2}}{3 s_{1}-s_{2}} .
$$

It can be seen that if $s_{1}=s_{2}=s$, then $R_{1}=-R_{2}=-2 s$, so that the radii of curvature are equal but with different signs, as expected. The angle between the optical axis and the rays between the two components is $\theta_{a}=\theta_{g 1}+\varphi_{1}=2 h / R_{1}+\left|h / s_{1}\right|$. Substituting for $R_{1}$ from (8) gives

$$
\theta_{a}=\frac{h\left(s_{1}-s_{2}\right)}{2 s_{1} s_{2}} .
$$

Eliminating $\varphi_{1}$ and $\varphi_{2}$ from (5), with equal grazing incidence angles, gives the grazing angle for this configuration

$$
\theta_{g}=\frac{h}{4} \frac{s_{1}+s_{2}}{s_{1} s_{2}}
$$

and the width $b$ of the transmitted beam is

$$
b=l \theta_{g}=\frac{l h}{4} \frac{s_{1}+s_{2}}{s_{1} s_{2}},
$$

noting that the latter result neglects reflections from the opposite sidewalls, that is, a nondiverging beam and $s_{1}, s_{2} \gg$ h.

So that the rays reflected from the first component are reflected by the second, the gap $D$ between them must be small enough such that the rays intersect the width $b$ in the second component, that is,

$$
D \ll \frac{l h}{4 \theta_{a}} \frac{s_{1}+s_{2}}{s_{1} s_{2}},
$$

which gives, by substituting for $\theta_{a}$,

$$
D \ll \frac{l}{2} \frac{s_{1}+s_{2}}{s_{1}-s_{2}} .
$$

If $s_{1}=s_{2}$, then the rays between the components are nominally parallel, and there is no limitation on the gap $D$ given by (13). In this case, the divergence of the rays set by the source distance $s_{1}$ becomes important, and we require $D \ll s_{1}$ so that rays which reflect from channels in the first component intersect with channel walls of the seconds component.

2.1.2. Flat-Curved Double-Reflection MOAs. With the first component unbent, and the second component bent, (5) are still valid, but now $\varphi_{1}=0$, corresponding to the required infinite radius of curvature. In addition, $\varphi_{2}=\theta_{g 1}+\theta_{g 2}=$ $h / R_{2}$, so that

$$
R_{2}=\frac{2 s_{1} s_{2}}{s_{1}+s_{2}}
$$

noting that $s_{1}=3 s_{2}$ gives $R_{1}=\infty$ for the equal grazing angle case, (8), and $R_{2}=s_{1} / 2$ for the flat-curved combination (14). Satisfying these two requirements simultaneously is a special case for which the first component is flat and the grazing angles are equal in the two components. For the flat-curved combination, $\theta_{a}=\theta_{g 1}$ and (13) are reduced to the simpler criterion that $D \ll l$ to avoid heavy losses.

2.1.3. Collimator-Telescope Double-Reflection MOAs. A collimator-telescope configuration requires $R_{1}=-2 s_{1}$ and $R_{2}=2 s_{2}$, where the negative sign indicates that the first component is curved with the centre of curvature on the object side so the two components curve in opposite directions. If $R_{1}$ is set correctly and the reflecting surfaces are flat and introduce negligible scatter, then rays in the gap between the components will be parallel to the axis and the gap can be widened to accommodate other optical elements, such as a grating, filters, and/or support structure without loss in performance.

2.2. Spot-to-Spot MOA Focusing Using Planar Channels. We can achieve spot-to-spot focusing with two-planar channel MOAs using the geometry described in Section 2.1, but setting the channels in the second component (aligned to, for example, the $y$ axis) are set perpendicular to those in the first (aligned to the $x$ axis). If there is a gap between the components (which there must be in any practical system), then the object and image distances, $s_{1}$ and $s_{2}$, will be slightly different for the first and second component, and hence the magnification will be slightly different in the $x$ and $y$ axes on the image plane, but otherwise the system will provide true imaging limited only by the resolution set by the width of the channels (see Section 2.3). The radii of curvature of the components can be set independently using (4) and the effective $f$-ratio of the optic will be as given in Section 2.1. In such an arrangement, there is no need for accurate alignment of the channels in the first component with those in the second, and the size of the gap $D$ is not critical and has little impact on the efficiency.

\subsection{Efficiency and Resolution}

2.3.1. Line-To-Line Focusing. The width $b$ of the beam transmitted through the channels increases linearly with axial offset $h$ until $b=w$, the width of the channel. At this offset, the opposite wall of the channel starts to block the inner edge of the beam. As $h$ is increased further, the beam width drops linearly until it reaches zero at the edge of the useful aperture which is given by (for a single-reflection MOA in the equal grazing angle case)

$$
h_{\max }=\frac{4 w}{l} \frac{s_{1} s_{2}}{s_{1}+s_{2}} .
$$

Note that $h_{\max }$ will be twice this value for a double-reflection MOA; using two reflections doubles the width of the available aperture. The mean beam width across the aperture is $b_{\text {mean }}=w / 2$. 
The geometric area of the single-reflection aperture which provides the line-to-line focusing is given by the sum of all the channels over a square aperture of size $W=$ $2 h_{\max }$. If the channels are evenly spaced with a period $p$, the geometric area is

$$
A_{\text {geom }}=\frac{32 w^{3}}{p l^{2}}\left(\frac{s_{1} s_{2}}{s_{1}+s_{2}}\right)^{2} .
$$

The effective area is limited by the grazing incidence reflectivity of X-rays from the channel walls. For a given Xray energy, there is a critical angle $\theta_{c}$ for reflection, that is, the grazing angle below which the reflectivity is high. Efficient use of the channels thus requires $w / l \approx \theta_{c}$, so that

$$
h_{\max }=4 \theta_{c} \frac{s_{1} s_{2}}{s_{1}+s_{2}} .
$$

So long as channels can be manufactured with this aspect ratio $(l / w)$ and over an area of $2 h_{\max } \times 2 h_{\max }$ then the maximum effective area which can be achieved at the X-ray energy corresponding to the critical angle is

$$
A_{\mathrm{eff}} \approx 32 \theta_{c}^{2} \frac{w}{p}\left(\frac{s_{1} s_{2}}{s_{1}+s_{2}}\right)^{2},
$$

assuming that the reflectivity is $100 \%$ for $\theta_{g}<\theta c$. This represents an idealised upper limit to the effective area for a single-reflection MOA. If we use a double-reflection MOA, $h_{\max }$ is increased by a factor of two because the grazing angles are reduced by a factor of two, and the areas $A_{\text {geom }}$ and $A_{\text {eff }}$ will be increased by a factor of four. In practice absorption and scattering will reduce the reflectivity, and the aspect ratio corresponding to this effective aperture will be hard to achieve; a silicon surface reflecting titanium $K_{\alpha} \mathrm{X}$-rays would require $l / w \approx 140$ while in the current work only $\approx 30$ has been achieved.

Planar channel walls provide no focusing. If the source line is very narrow, the width of the on-axis line from a single channel is

$$
\delta=\frac{l h s_{2}}{4}\left(\frac{s_{1}+s_{2}}{s_{1} s_{2}}\right)^{2},
$$

so that the best resolution (minimum $\delta$ ) will result from short channels close to the optical axis but these channels provide very little effective area. If the aperture is constructed to give the maximum area as described above then the resolution from the combination of channels across the aperture is determined by the average effective beam width $w / 2$. A finite source size $\chi$ will increase the line focus width by $\chi s_{2} / s_{1}$ so that the overall line focus width for a singlereflection configuration $\Delta_{1}$ is the combination of two terms,

$$
\Delta_{1} \sim\left(\frac{w}{2} \frac{s_{1}+s_{2}}{s_{1}}, \chi \frac{s_{2}}{s_{1}}\right) ;
$$

if the profiles are Gaussian, $\Delta_{1}$ is given by adding the two terms in quadrature but for other profiles the combination will have a different form.

The centre of the line-spread distribution corresponds to rays which reflect from half way down the channel walls while the extremities correspond to rays which reflect from the ends. Because the channel walls are planar and not curved, the reflections from the ends introduce a small angular reflection error $\Delta \theta_{\max } \approx w / s_{1}$. This maximum reflection angle error occurs for $h=h_{\max } / 2$. For a double-reflection configuration the maximum reflection angle error is given by the same expression but this now occurs at twice the axial offset, $h=h_{\max }$, because the radii of curvature of the plates is twice as large. However, each ray in the double-reflection case suffers two reflection errors so the line-spread function contribution from the length of the channels will be twice as wide.

$$
\Delta_{2} \sim\left(w \frac{s_{1}+s_{2}}{s_{1}}, \chi \frac{s_{2}}{s_{1}}\right) .
$$

For many applications, it is useful to express the performance of a device in terms of a gain given by the ratio of the geometric (or effective) aperture area to the area of the line (or spot) focus. If we use the first term in (20) for the focus width and the total length of the line focus as $4 h_{\max }$ (this length is twice the width of the MOA aperture) then the geometric gain of a single-reflection device is

$$
G_{\text {geom }}=\frac{4 w}{p l} \frac{s_{1} s_{2}^{2}}{\left(s_{1}+s_{2}\right)^{2}} .
$$

The gain of a double-reflection configuration will be twice this because the aperture area is increased by a factor of four while the line-spread width is increased by a factor of two. Of course, the true gain will be limited by the $\mathrm{X}$-ray reflectivity and scattering losses, so $G_{\text {geom }}$ represents an idealised upper limit.

In summary, the maximum aperture half width, geometric area, and width of the line-spread function for a singlereflection MOA are given by (15), (16), and (20), respectively. For a double-reflection MOA, the maximum aperture width is twice that of a single-reflection MOA. This increases the geometric area by a factor of four and the line-spread width by a factor of two. Therefore the flux per unit area in the line-spread function from a double-reflection MOA could be twice that of a single-reflection MOA. That is, the doublereflection system has twice the gain. However, it is likely that channel alignment, figure errors in the channel walls and surface roughness of the channel walls will limit the performance of a double-reflection device, and this potential advantage over a single-reflection MOA may not be realised.

Adjustment of the curvature of the plates gives us potential control over the focused line width and, for a doublereflection MOA, the flux level in the focused line. For a single-reflection device, we can tweak the curvature, $R$, using piezoelectric actuators, so that it matches the requirement given by (4). In principle, an array of actuators could be employed such that any error in the bending or deviations away from the cylindrical (circular) form could be reduced or eliminated. For a double-reflection configuration tweaking the curvature of the first plate will alter the convergence or divergence of the beam (rays) in the gap between the plates. This will provide control over the flux that intersects with the channels in the second plate and hence the flux in the focused 
line/spot. Tweaking of the curvature of the second plate then gives us control of the size of the focused line, but this has only a very small effect on the efficiency.

For the double-reflection configuration, the lateral and rotational alignment of the two elements is also critical in achieving the highest efficiency. It is envisaged that these alignments would have to be adjusted in conjunction with the curvature of the first plate to realise the highest flux level in the focused line. The potential flux advantage of the double-reflection device would only be achieved if such adjustment was possible/successful.

2.3.2. Spot-to-Spot Focusing. For a crossed pair of planar channel MOAs in the arrangement described in Section 2.2 the geometric area is a factor of $w / 2 p$ less than a singlereflection MOA because only that fraction of the flux focused by the first component is intercepted by the channels of the second. The geometric aperture area is therefore

$$
A_{\text {geom }}=\frac{16 w^{4}}{p^{2} l^{2}}\left(\frac{s_{1} s_{2}}{s_{1}+s_{2}}\right)^{2},
$$

where we have assumed that the gap between the components is small such that $s_{1}$ and $s_{2}$ are the same for both. Two reflections give focusing in two axes so the area of the focused spot (assuming a point source) is

$$
\Delta_{1}^{2}=\frac{w^{2}}{4}\left(\frac{s_{1}+s_{2}}{s_{1}}\right)^{2} .
$$

Therefore the geometric gain is given by

$$
G_{\text {geom }}=\frac{64 w^{2}}{p^{2} l^{2}} \frac{s_{1}^{4} s_{2}^{2}}{\left(s_{1}+s_{2}\right)^{4}},
$$

and the ratio of the gain for the spot-to-spot focusing using a crossed pair compared with line-to-line focusing using just one $\mathrm{MOA}$ is $2 w s_{1} /(p l)$.

In order to achieve the optimum (minimum spread) focus in a crossed pair configuration, we required adjustment of either both the radii of curvature of the components or the adjustment of the radius of one component and the position of the detector or source along the axis (tweak of $s_{1}$ or $s_{2}$ ). These adjustments could be provided using piezoelectric actuators and would give independent control of the focus in $x$ and $y$ directions on the image plane.

2.4. Circular-Channel MOAs. A schematic of the geometry of a two-reflection MOA with circular channels is shown in Figure 3. This is similar to the flat-curved configuration with planar channels considered above except that the two components are set with separation (of the centres along the axis) $D$; the grazing angles on the first and second components are $\theta_{1}$ and $\theta_{2}$; distances $h_{1}$ and $h_{2}$ now become radii of the reflecting surface walls, $r_{1}$ and $r_{2} ; S$ is the distance from the source to the first component; $d$ is the axial distance between the centre and edges of the second component (introduced by the curvature); $F$ is the focal distance measured from the centre of the second component which is bent to a radius $R$. We have

$$
\begin{gathered}
\tan \theta_{1}=\frac{r_{1}}{S}, \\
r_{2}=r_{1}-(D+d) \tan \theta_{1}, \\
(R-d)^{2}+r_{2}^{2}=R^{2} .
\end{gathered}
$$

Assuming that $D$ and $R$ are set by the user, we can solve (26) giving $d$ and $r_{2}$ for a given $r_{1}$. Then, $\theta_{2}$ can be calculated from

$$
\begin{aligned}
& \tan \left(\theta_{1}+\theta_{2}\right)=\frac{r_{2}}{(R-d)}, \\
& \tan \left(\theta_{1}+2 \theta_{2}\right)=\frac{r_{2}}{F-d},
\end{aligned}
$$

These equations hold for rays which come from a point source on axis and reflect from the centres of the channels. In practice, radiation incident on the first component before (after) the centre will have a larger (smaller) grazing incidence angle and will therefore hit the second component channel after (before) the centre. The result is a broadening of the focal spot. The system provides point-to-point imaging with a resolution limited by the length of the channel wall, $l$. The rays diverging from the source intersect the rays converging to the focus at a principal surface which is approximately planar and lies between the first and second components, perpendicular to the optical axis. Thus a source spot of finite size will be imaged to a focal spot of finite size with demagnification $\approx F / S$.

The geometric aperture area and gain of a two-reflection MOA with circular channels could be comparable to or larger than a crossed pair of planar channel devices as described in Section 2.3.2 but it would be critically dependent on the manufacture tolerances of the channels and the coalignment of the two components. Circular channel MOAs can be aligned and adjusted for optimum performance in the same way as planar channel devices. The spot flux and spot size produced by the flat-curved configuration illustrated in Figure 3 could be controlled by changing both the separation, $D$, and curvature, $R$, using piezo actuators.

\section{Manufacture of MOAs}

There are two stages in the manufacture of the types of optics discussed in the previous section: first, to produce the channel structures and second, to bond the actuators. To date, only the 1-D planar channel components have been made.

3.1. Deep Silicon Etching of the Channel Structures. The channel structures of the MOAs can be formed by silicon etching via the Bosch process [13], using a pattern mask and a cycle of etching/passivation. The gases used are $\mathrm{SF}_{6}$ for etching and $\mathrm{C}_{4} \mathrm{~F}_{8}$ for passivation; the latter prevents sidewall etching and so allows deep channels with parallel walls to be made. However, the cyclic etch process results in quasi periodic "terracing" or "scalloping" of the sidewalls as the 
etch proceeds down into the silicon. Also, line edge roughness on the mask-which is random but not periodicis replicated on the etched sidewalls as vertical striations, the "curtain effect". Both of the surface roughness features are illustrated by the SEM pictures shown in Figure 8. They must be minimised to prevent excessive X-ray scattering. A root-mean-square roughness of around $2 \mathrm{~nm}$ is required as discussed in the next section. Early etching attempts did not provide roughnesses much better than $1 \mu \mathrm{m}$, but a succession of changes to the process cycle has led to values within an order of magnitude or so of those required, coupled with deep channels and relatively straight walls. The changes include the following:

(i) shortening of the etch/passivate cycle time to reduce both the amplitude and the spacing (wavelength) of the scallops,

(ii) the use of "over-run", that is, overlapping the etch and passivate stages, to smooth the peaks and troughs of the scallops,

(iii) varying the gas flow rates and pressure,

(iv) following etching, the channel walls are subject to oxidisation followed by removal, which has the effect of "snapping off" the peaks.

Using such improvements, other authors have achieved sidewall roughnesses of $\sim 10 \mathrm{~nm}$ [14]; although this is still several times larger than that required, it is likely that further changes to the processes can provide further improvements. An alternative to the "dry" etching process is "wet" etching whereby the silicon is immersed in a chemical solution and is dissolved to achieve the desired shape by the use of a mask. This preferentially etches along the $\langle 111\rangle$ or $\langle 110\rangle$ silicon crystal planes, creating very smooth, straight walls. Initial testing indicates that the surface roughness achieved is much lower than that for the dry etching process, providing that the mask edges are accurately aligned to the crystal lattice, but this technique cannot be used to produce cylindrical geometry because the etch follows the crystal planes. A full analysis of the manufacturing process will be given in subsequent publications.

3.2. Actuator Control. Several $2 \times 1 \mathrm{~cm}$ silicon chips with $2 \times$ $2 \mathrm{~mm}$ active areas have been epoxy-bonded to piezo actuators with the general arrangement shown in Figure 7, and these are awaiting testing. Issues that are being addressed include piezo thickness, and width, bonding agent thickness and the use of low-shrinkage glues (to prevent shrinking itself causing bending). Different types of actuators, including unimorph (as discussed here), bimorph, and piezo fibres are also being considered. These aspects will also be discussed in future publications.

\section{X-Ray Scattering from the MOA Walls}

Surface roughness in the axial direction (across the scallops) will cause in-plane scattering while the curtain profile will give either out-of-plane reflection errors and/or out-of-plane

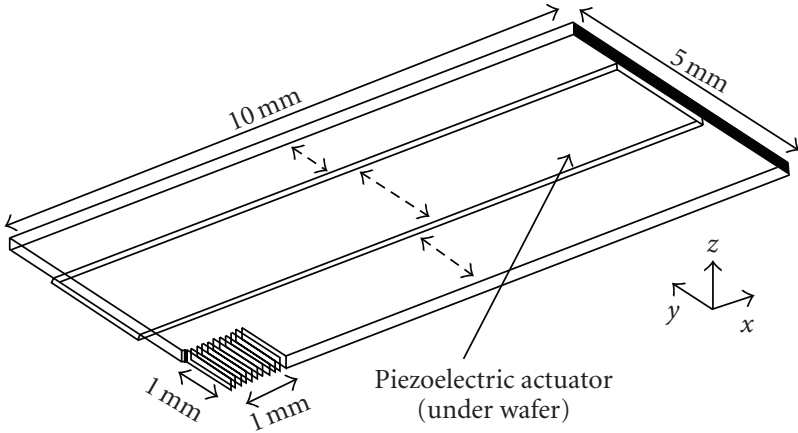

FIGURE 7: Schematic of the piezo actuator bonded to silicon wafer. A quarter of a 1-D chip is shown. The bottom left corner contains the etched channels which cover $2 \times 2 \mathrm{~mm}^{2}$ in the centre of the chip. The dashed line indicates dimensions which were varied in the FEA modelling.

scattering in which the curtain behaves like a ruled grating in a conical diffraction configuration.

4.1. In-Plane Scattering. Figure 9 shows the geometry of inplane scattering from the axial surface roughness across the scallops. This scattering is conventionally calculated using first-order theory which gives the Total Integrated Scatter (TIS),

$$
\text { TIS } \simeq\left(\frac{4 \pi \theta_{g} \sigma}{\lambda}\right)^{2}
$$

where $\sigma$ is the rms surface roughness; $\lambda$ is the X-ray wavelength; $\theta_{g}$ is the grazing angle. This will be a good approximation provided that $\theta_{g} \sigma / \lambda \ll 1$ and the TIS is a few percent. Rearranging gives

$$
\sigma \simeq \frac{\lambda(\mathrm{TIS})^{1 / 2}}{4 \pi \theta_{g}} .
$$

If we take $\lambda=10 \AA(1.24 \mathrm{keV}), \theta_{g}=2 \times 10^{-2}$ radians $(1.14$ degrees) then $\sigma=4(\mathrm{TIS})^{1 / 2} \mathrm{~nm}$, and we require $\sigma \simeq 2 \mathrm{~nm}$ to keep the TIS small $(<0.25)$ as already stated above. The scattering angles will be given by the grating equation

$$
\frac{n \lambda}{d \sin \theta_{g}}=\sin \beta,
$$

where $d$ is the correlation length of the surface roughness along the axial direction down the scallop. If the surface is much rougher than this, then higher-order scattering and/or shadowing (absorption) will become important and TIS $\rightarrow$ 1. The higher orders can, in principle, be calculated but we require detailed knowledge of the statistical properties of the surface height distribution over and above $\sigma$ and $d$.

4.2. Out-Of-Plane Reflection/Scattering. The walls of an MOA will act as a diffraction grating with the grating spacing given by the width of the curtain features $b$ as shown in 


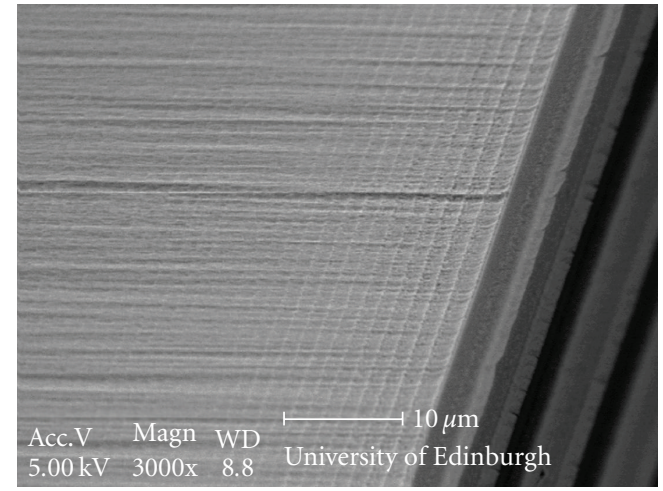

(a)

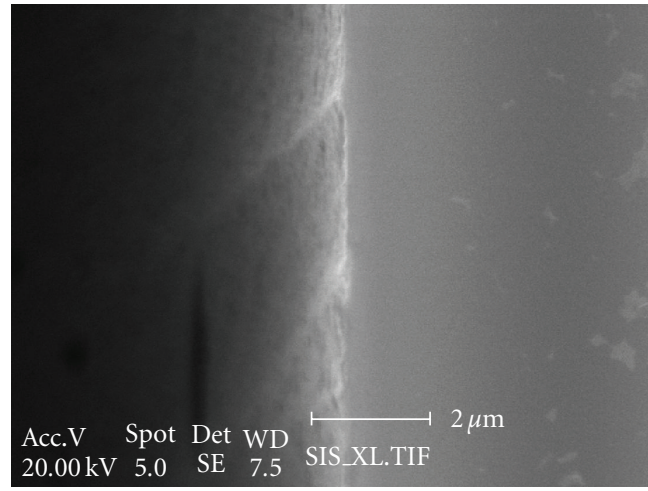

(b)

FIGURE 8: (a) Scalloping on the etched channel walls. (b) Details of the curtain effect caused by rough edges of the etching mask.

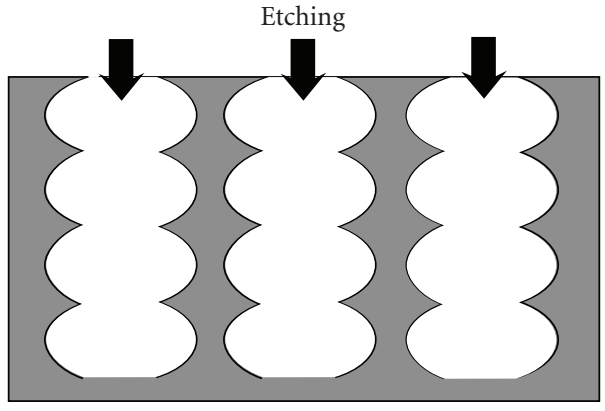

Active area channels

(a)

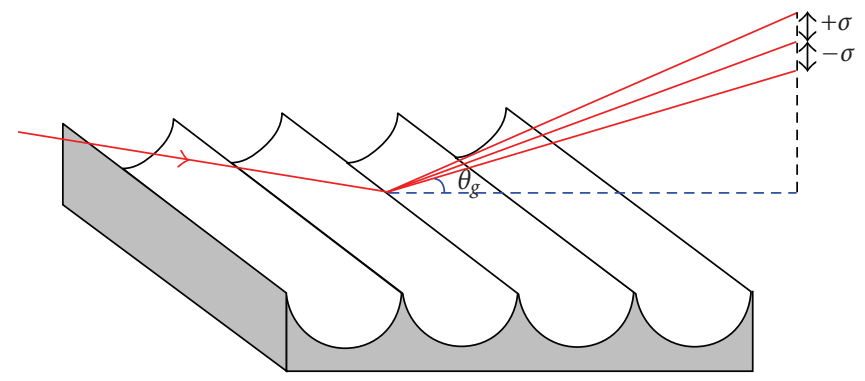

(b)

Figure 9: In-plane scattering from the MOA wall with scallops of width $b$, height $h$, and radius $T$.

Figure 10. The grating equation gives a diffraction angle outof-plane

$$
\sin \beta_{d}=\frac{\lambda}{b \sin \theta_{g}},
$$

which for small angles reduces to

$$
\beta_{d}=\frac{\lambda}{b \theta_{g}} .
$$

Taking the curtain profile to be a sector of a circle, the radius of the profile can be calculated using the height of the curtain features, $h$, and the width, $b$,

$$
T \simeq \frac{b^{2}}{8 h} .
$$

Using the radius of the curtain profile $T$ and the spacing between the cusps $b$, the maximum out-of-plane gradient error is then given by

$$
\Delta g_{\max }=\frac{b}{2 T},
$$

and the average gradient error is then

$$
\overline{\Delta g}=\frac{b}{4 T} \text {. }
$$

Therefore, the average out-of-plane deflection error (twice the gradient error) from the curtain is

$$
\beta_{r}=\frac{2 b}{4 T}=\frac{b}{2 T} .
$$

The dividing line between geometric optics (figure error dominated) and wave optics (scattering and/or diffraction dominated) is given by equating the two angles, $\beta_{d}$ and $\beta_{r}$, giving

$$
\frac{b}{2 T}=\frac{\lambda}{b \theta_{g}},
$$

and rearranging to give

$$
\frac{b^{2}}{T}=\frac{2 \lambda}{\theta_{g}} .
$$

If $b^{2} / T$ is larger than this (i.e., $T$ is small or $b$ is large) then the geometric out-of-plane reflection errors dominate. If $b^{2} / T$ is smaller than this (i.e., $T$ is large or $b$ is small) then the diffraction from the scallops dominates. $b$ and $T$ (and therefore $h$ ) will be fixed by the channel manufacture, and $\lambda$ will be restricted to a given range so the dominant varying factor will be $\theta_{g}$. This will be small for the channels 


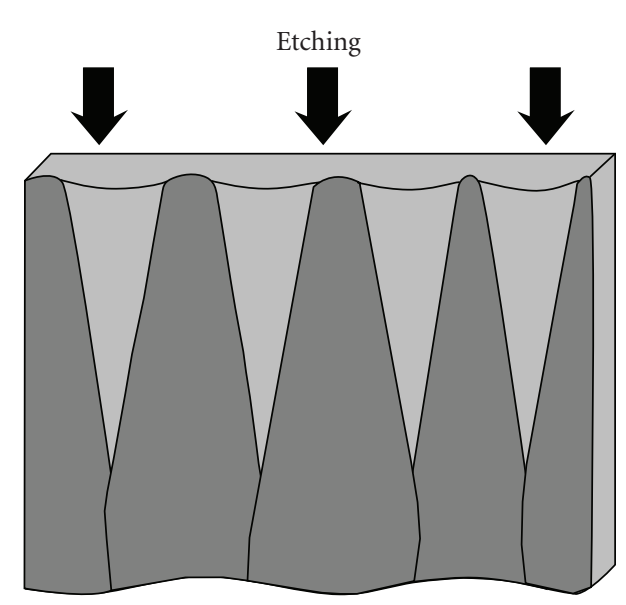

(a)

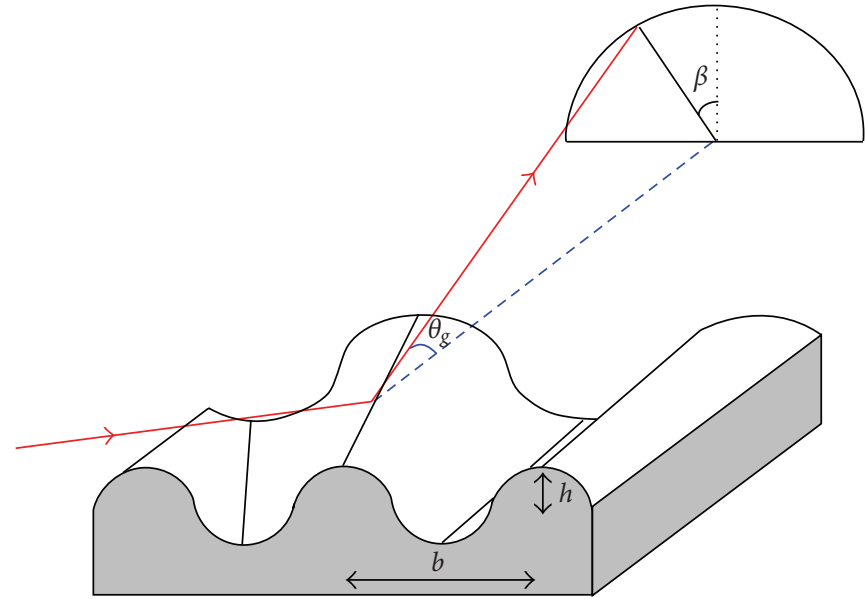

(b)

Figure 10: Out-of-plane scattering of an MOA wall with the curtain effect.

at the centre of the MOA plates and large at the edges. Again rearranging and using (33), (37) becomes

$$
\theta_{g}=\frac{\lambda}{4 h}
$$

thus giving us the dividing line between geometric reflection errors and diffraction over the MOA aperture.

We can look at this from the point of view of the 1storder scattering theory. For the out-of-plane diffraction, the TIS is dependent on the height of the scallops, $h$, just as the in-plane TIS is dependent on the rms surface roughness, $\sigma$. The out-of-plane TIS from the scallops is given by

$$
\mathrm{TIS}=\left(\frac{4 \pi \theta_{g} \sigma_{h}}{\lambda}\right)^{2}
$$

where $\sigma_{h}$ is the rms height of the scallops. If $h \ll T$, then we can we can estimate this rms height

$$
\sigma_{h}=\sqrt{\overline{h^{2}}-\bar{h}^{2}}
$$

giving

$$
\sigma_{h}=\frac{2 h}{3 \sqrt{5}}
$$

Substituting this into (44), setting TIS $=1$, and rearranging, we get

$$
\theta_{g}=c \frac{\lambda}{4 h}
$$

where

$$
c=\frac{3 \sqrt{5}}{2 \pi}=1.068 .
$$

This is comparable to the result produced in (39)and again gives the dividing line between geometric reflection errors and scattering/diffraction. At small grazing angles the phase errors introduced by the height error of the scallops are small, so scattering drops and geometry take over. As $\theta_{g}$ increases, the height errors become important and at some point scattering takes over. This also means that diffraction dominates when the projected area of the channel walls is large.

The typical curtain width is $b=800 \mathrm{~nm}$. Assuming $\lambda=$ $10 \AA$ and $\theta_{g}=2 \times 10^{-2}$ radians, (32) gives a diffraction angle of $\beta_{d}=0.63$ radians or 35 degrees. When the depth of the curtain, $h$, is given by (39) (or (44)), the deflection error $\beta_{r}$, given by (36), will also be 35 degrees. If we are using planar channels to produce a line focus, this out-of-plane deflection will simply shift rays out-of-plane, and there should be little effect on the focus but there will be a marked decrease in the efficiency. Using the same wavelength and grazing angle we require a curtain depth $h \ll 50 \mathrm{~nm}$ to prevent excessive losses due to out-of-plane scattering/reflection. For a crossed planar or circular-channel double-reflection configuration which can produce a focused spot rather than a line focus, the out-of-plane scattering by the curtain is a more serious problem. The curtain depth must be reduced to $h \approx 6.7 \mathrm{~nm}$ so that $\sigma_{h} \approx 2 \mathrm{~nm}$ and out-of-plane scattering is reduced to the same level as the in-plane scattering.

\section{Finite Element Analysis and Ray-tracing}

The MOA channels are made in flat silicon and then bent using piezo actuation to give the required focusing and active control. We are using finite element analysis (FEA) to model the effects of the bending on the channel walls, and the results from this will be fed into ray-tracing analysis in order to determine the properties of the MOA focus. Section 5.1 describes the progress to date on the FEA, and Section 5.2 discusses the results of ray-tracing. The latter uses in-house software as commercial packages are not well suited to MOA structures (Zemax was used for the same inititial ray-tracing 


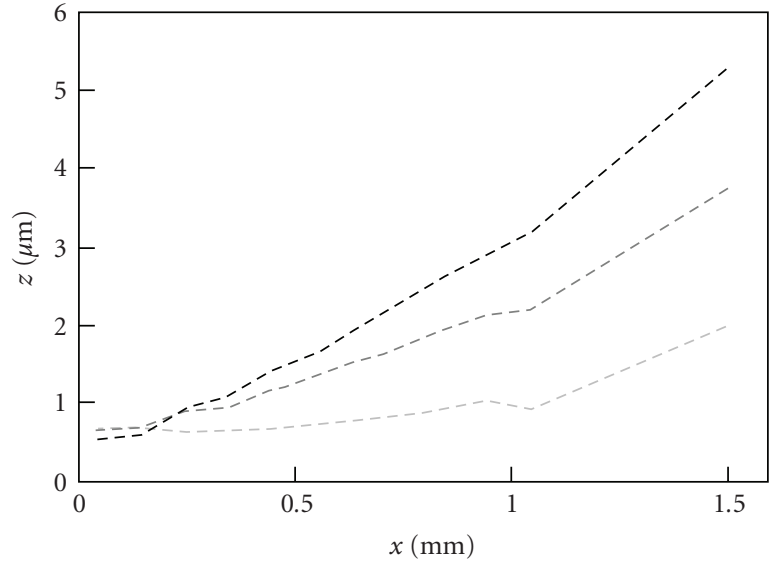

(a)

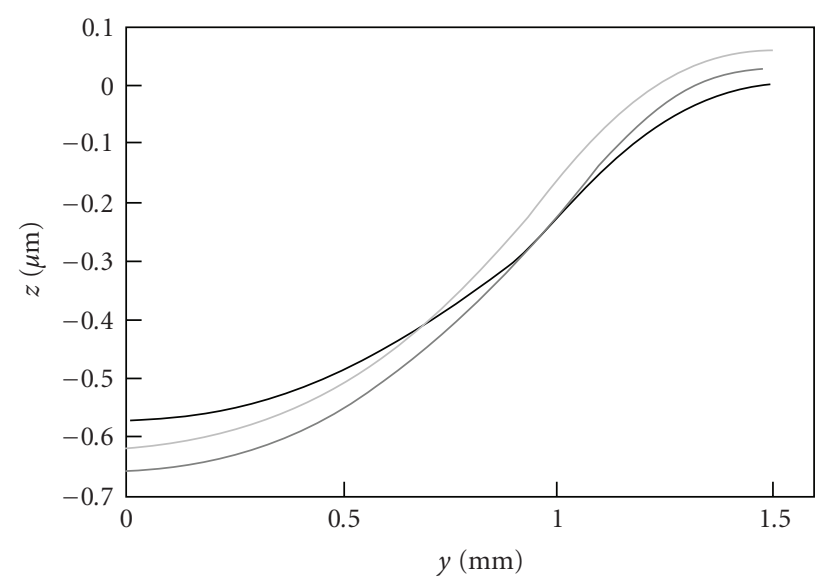

(b)

FIGURE 11: FEA results for bending of a 1-D MOA using two strips of $150 \mu \mathrm{m}$ thick PZT-5H. See text for details.

analysis but this was very cumbersome and time consuming). The FEA results have not yet been incorporated in the raytracing.

5.1. Finite Element Analysis. The FEA was carried out using the commercial package COMSOL Multiphysics [15]. The aim was to study the stress and displacement of a 1-D MOA when bent by a unimorph piezoelectric actuator in different configurations. Only a quarter of the silicon wafer was modelled to take advantage of the symmetry, thus reducing the processing time and allowing finer FEA meshing. The geometry is shown in Figure 7. Figure 11 shows the bending produced by two strips of $150 \mu \mathrm{m}$ thick PZT-5H placed adjacent to the active area. The dark curve is for strips $4 \mathrm{~mm}$ wide, that is, covering the whole area between the active area and the edge of the silicon chip (see Figure 7). The grey curve is for $2 \mathrm{~mm}$ wide strips and the light curve for $1 \mathrm{~mm}$ wide strips. In these calculations, the effect of glue used to bond the actuators was neglected. The applied voltages were the maximum that the piezos are expected to withstand, namely $\sim 2.5 \mathrm{~V} / \mu \mathrm{m}$ of thickness. The curvature required is along the $\mathrm{X}$-axis. Note that there is a small step at $x=1 \mathrm{~mm}$ which corresponds to the edge of the etched area which contains the channels. Clearly, the bending results in very complex shapes, and more detailed analyses are ongoing. The smallest radius of curvature, produced by the $4 \mathrm{~mm}$ wide strips of PZT-5H, is $\approx 200 \mathrm{~mm}$. Similar curves produced using piezo thicknesses of $50 \mu \mathrm{m}$ and $100 \mu \mathrm{m}$ indicate that this is the smallest radius of curvature which can be produced with PZT-5H. The typical radius of curvature required for optimum 1-D single-reflection designs is $\sim 50 \mathrm{~mm}$ and for double-reflection configurations $\sim 100 \mathrm{~mm}$ although some configurations in which $s_{1} \approx s_{2}$ utilize flat plates or very large radii. Further work is required to find the optimum piezo material and geometry which can generate and give adequate adjustment of these curvatures.

5.2. Ray-tracing. For the ray-tracing described in this section, the software "Q", developed at the University of
Leicester specifically for grazing incidence [16], was used. This is much better suited to optics such as MOAs than commercial packages are and has the flexibility to allow future add-ons, for example, to take diffraction into account. In the examples given here the rays travel from right to left. In all the simulations presented, the same channel dimensions were assumed; channel width $w=10 \mu \mathrm{m}$, channel pitch $p=20 \mu \mathrm{m}$, and channel depth (axial length) $l=200 \mu \mathrm{m}$. In the first four cases, the total distance between the source and image plane $\left(s_{1}+s_{2}\right)$ was set to $40 \mathrm{~mm}$, and the aperture dimension was set to the appropriate value of $2 h_{\max }$ depending on the configuration. As a first example, line-toline focusing by a single-reflection MOA with $s_{1}=25 \mathrm{~mm}$, $s_{2}=10 \mathrm{~mm}$, and bending radius $R=33 \mathrm{~mm}$ is shown in Figure 12. The rays which suffer from one reflection and are brought to a line focus are shown in green. Rays which are not reflected but penetrate the channels near the axis and rays, towards the edge of the aperture, which suffer from two reflections are shown in red and blue, respectively. The width and integral of the focused line profile shown in the righthand panel are in good agreement with the predictions given by (20) and (16). The Full Width Half Maximum (FWHM) of the line profile is $16 \mu \mathrm{m}$ and the geometric area of the utilized aperture is $3.5 \mathrm{~mm}^{2}$.

Ray-tracing of a double-reflection equal grazing angle MOA is shown in Figure 13. This has $s_{1}=s_{2}=20 \mathrm{~mm}$ so that it is also a special case of the collimator-telescope configuration. The gap between the components is set to $2 \mathrm{~mm}$ so that rays in the gap, parallel to the axis, are clearly visible. The bending radii are $R_{1}=R_{2}=40 \mathrm{~mm}$, with opposite curvatures. Near the axis a few rays miss both components. Further out rays which reflect from the 1st component but miss the 2 nd are clearly visible as rays running parallel to the axis on the left. There are also a few rays which miss the 1st and reflect from the 2nd being brought to a focus half way between the 2 nd component and the image plane. The focused line profile in the right-hand panel is much broader than the single-reflection configuration shown in Figure 12 . This is partly because there are two reflections 


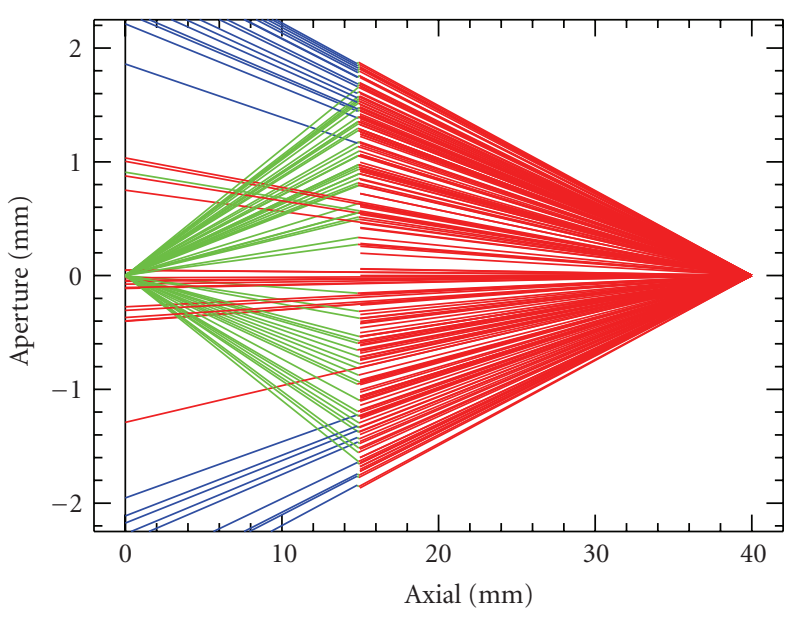

(a)

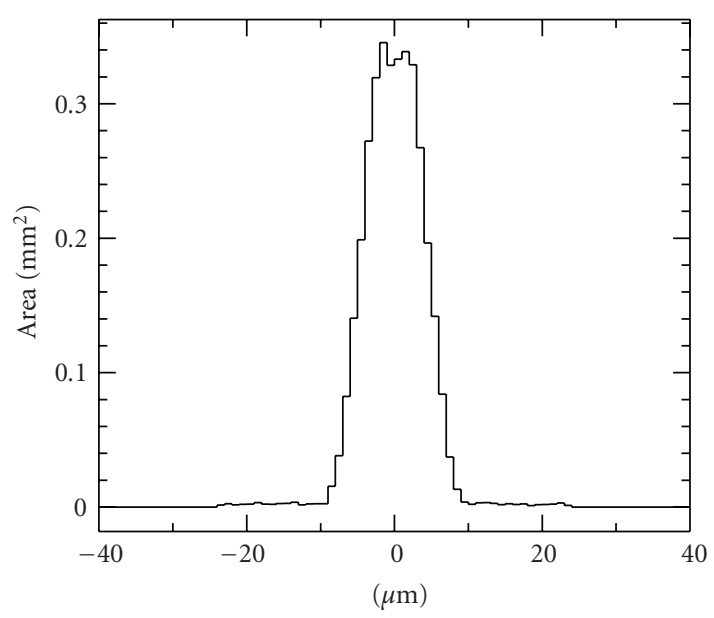

(b)

FIGURE 12: Line focusing by a single-reflection MOA (a) traced rays; red: no reflection, green: 1 reflection, blue: 2 reflections, (b) the focused line profile.

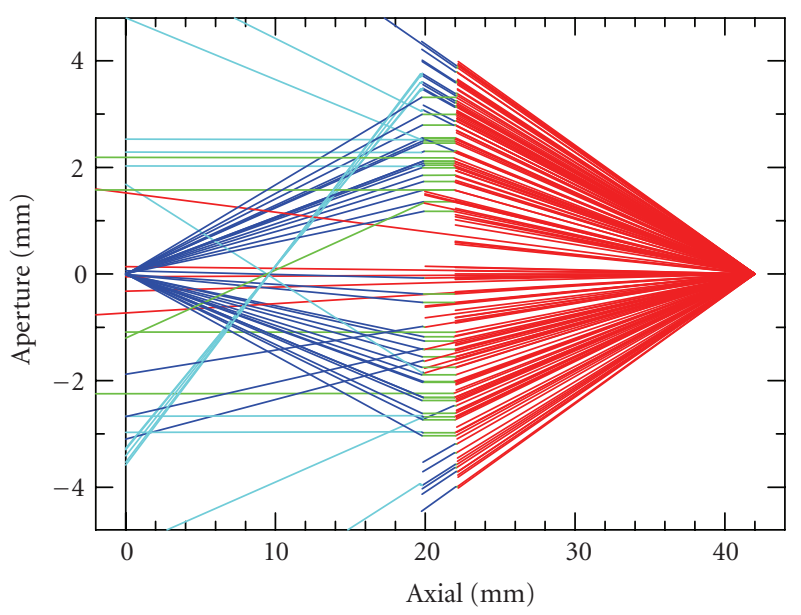

(a)

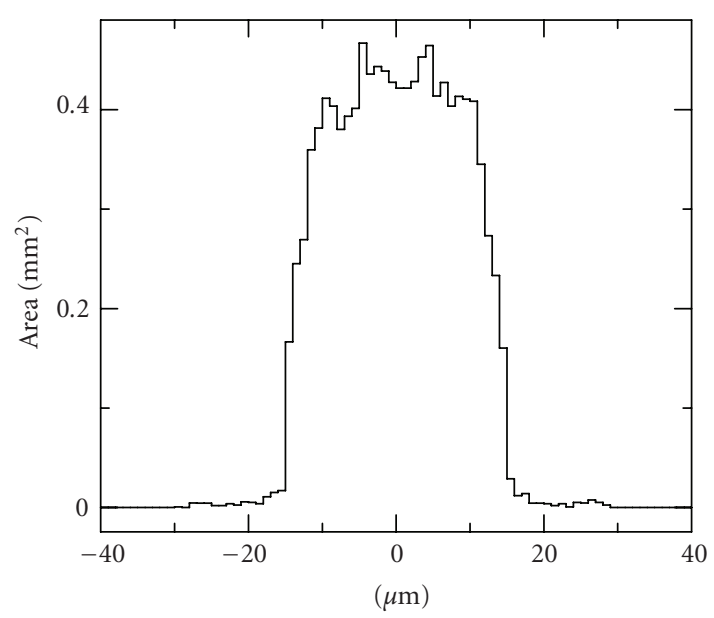

(b)

FIGURE 13: Line focusing by a double-reflection MOA with equal grazing angles. (a) Traced rays; red: no reflection, green: 1 reflection, blue: 2 reflections, (b) The focused line profile.

but also because $\left(s_{1}+s_{2}\right) / s_{1}=2$ whereas this ratio was set to 1.6 in the single-reflection case. The FWHM of the line profile is $30 \mu \mathrm{m}$. The effective aperture width and hence the integrated flux in the line-spread distribution is a factor 3.2 larger for the double-reflection configuration as expected; the geometric area of the utilized aperture is $11.1 \mathrm{~mm}^{2}$. In this configuration the double-reflection MOA provides a flux advantage but degrades focusing.

Figure 14 shows the ray-tracing results for a doublereflection flat-curved configuration with equal grazing angles. The structure in the focused line profile seen outside the central peak is due to rays which have penitrated both components without reflection. These could be eliminated using a small central aperture stop. As explained in Section 2.1.2 $s_{1}=3 s_{2}=30 \mathrm{~mm}$ and the curvature of the second plate must be $R_{2}=s_{1} / 2=15 \mathrm{~mm}$. In order to maximise the throughput, the gap was adjusted until the integral in the line-spread function was maximised. This corresponded to $D=0.45 \mathrm{~mm}$. The position of the source and the image plane were then tweaked to minimize the width of the line-spread function. The geometric area and line-spread function width for this configuration are very similar to the single-reflection case shown in Figure 12. The FWHM of the line profile is $9 \mu \mathrm{m}$, and the geometric area of the utilized aperture is $2.9 \mathrm{~mm}^{2}$ so, surprisingly, the raytracing simulation indicates that using two reflections in a flat-curved configuration gives very little performance benefit over the single-reflection configuration. This is because some flux reflected from the first plate is not intercepted by the channels in the second plate even when the gap has been optimised. Furthermore, experience in setting up the ray-tracing and optimizing the simulation performance indicates that the double-reflection configuration would be more difficult to align and adjust in reality. 


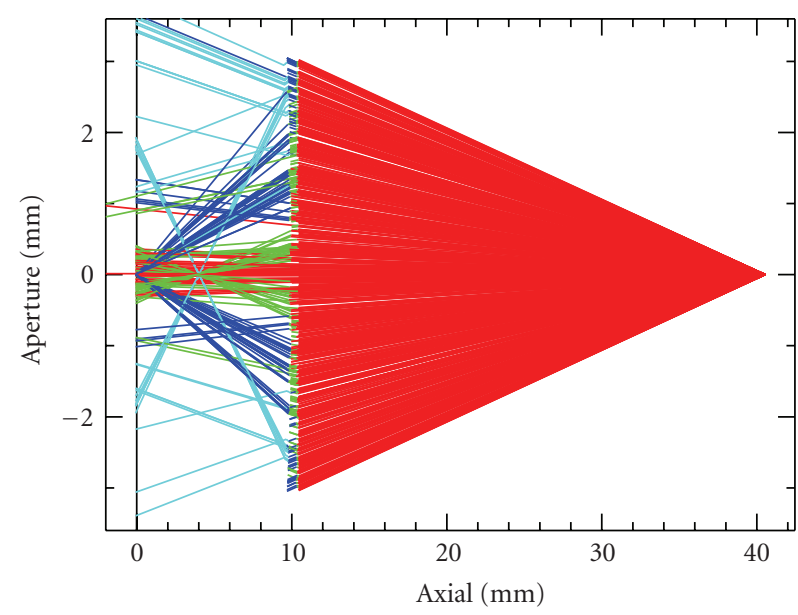

(a)

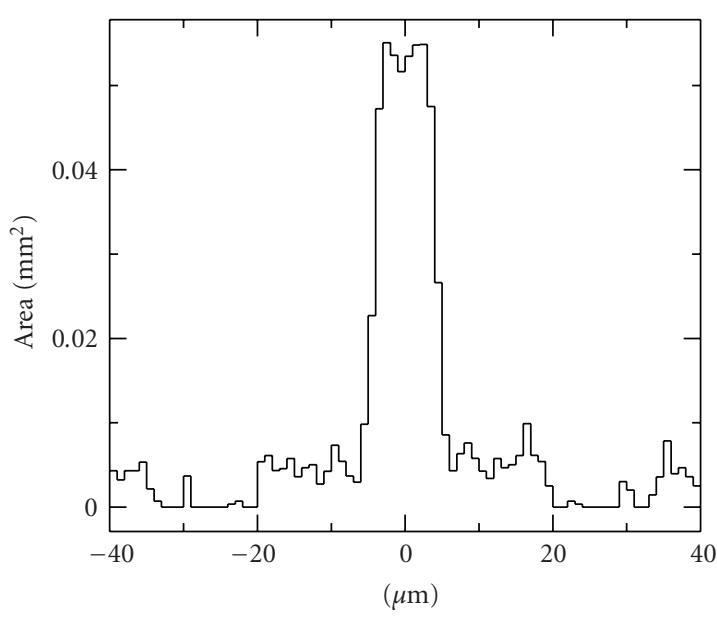

(b)

FIgURE 14: Line focusing by a double-reflection MOA with a plane-curved equal grazing angle configuration. (a) Traced rays; red: no reflection, green: 1 reflection, blue: 2 reflections, (b) The focused line profile.

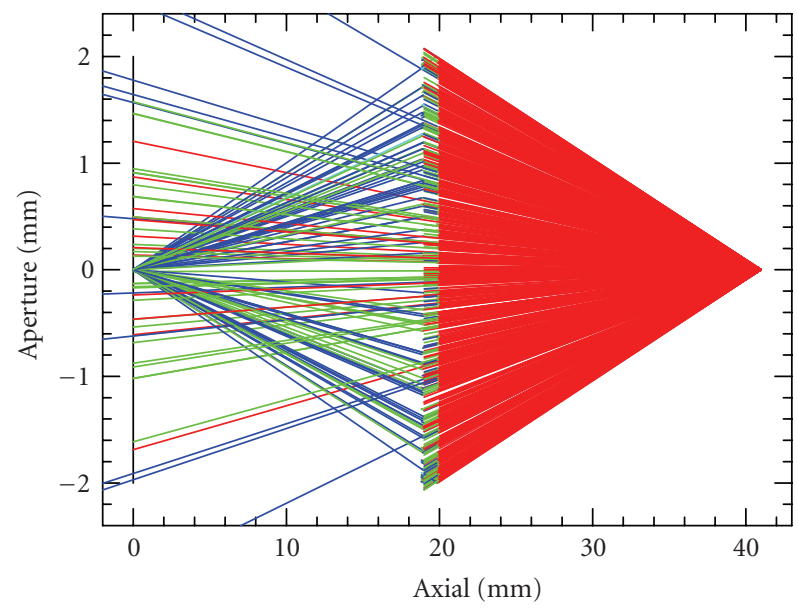

(a)

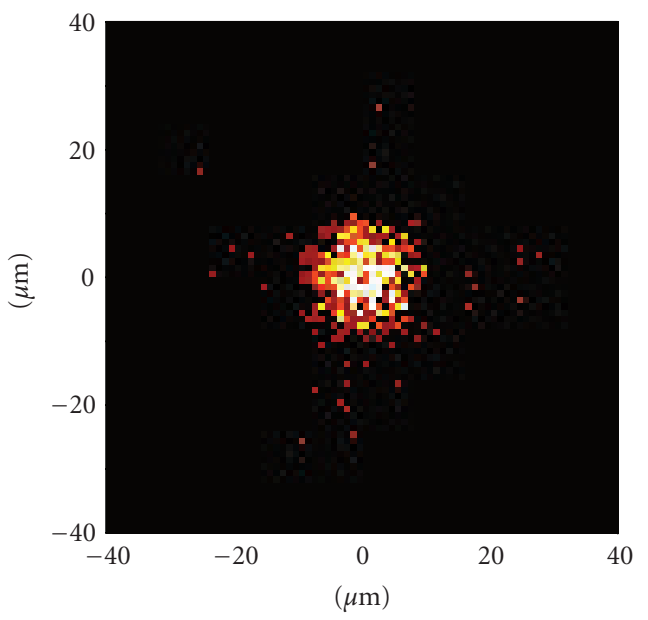

(b)

Figure 15: Spot focusing by a double-reflection crossed pair. (a) Traced rays; red: no reflection, green: 1 reflection, blue: 2 reflections. (b) Image of the focused spot.

Figure 15 shows a ray-tracing of a crossed doublereflection configuration. The first component is placed with $s_{1}=20 \mathrm{~mm}$ and the second with $s_{1}=21 \mathrm{~mm}$ so the gap is $D=1 \mathrm{~mm}$. The radii of curvature were adjusted in an iterative process to find the minimum spot Half Energy Width (HEW); $R_{1}=691 \mathrm{~mm}$ and $R_{2}=262 \mathrm{~mm}$. Thus the ray-tracing software was used to simulate the active adjustment of the components to optimise the performance. The geometric aperture area was $0.8 \mathrm{~mm}^{2}$, and the minimum HEW found was $10.1 \mu \mathrm{m}$. The FWHM of the spot was $9.1 \mu \mathrm{m}$.

Table 1 Summarises the ray-tracing results for the four configurations tested. The ray-tracing was run with perfect reflectivities to give the geometric area $A_{\text {trace }} \mathrm{mm}^{2}$ and the line/spot width FWHM $\mu \mathrm{m}$. It was then rerun using theoretical reflectivities as a function of grazing angle for
$1 \mathrm{keV}$ X-rays from a Silicon surface and Gold surface so that gains, $G_{\mathrm{Si}}$ and $G_{\mathrm{Au}}$, could be calculated. These represent the best estimates of the gains which could be achieved if the channel walls were perfectly smooth. The gains calculated using the simple formula, $A_{\text {geom }}$, and the ray-tracing, $A_{\text {trace }}$, are in reasonable agreement. The differences are due to a combination of losses not included in the formulae for double-reflectivity MOAs and a better estimate of the true width of the focused distribution is provided by the raytracing. The $G_{S i}$ values are all much lower because the critical angle from Silicon at $1 \mathrm{keV}$ is much smaller than that required to utilise the edges of the aperture. The $G_{A u}$ values are significantly better because the high- $Z$ material provides a much higher soft X-ray reflectivity than bare Silicon. For applications where the flux per unit area in the focused line/spot is paramount the crossed spot-to-spot 


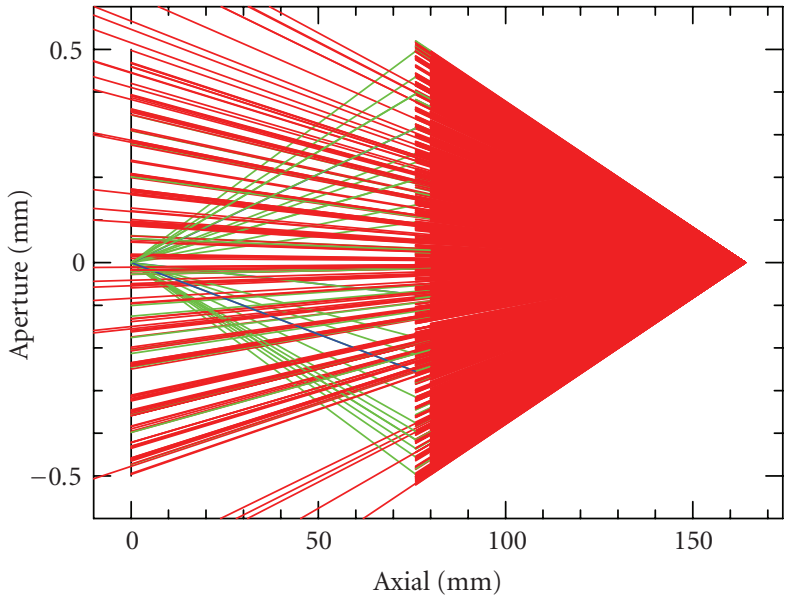

(a)

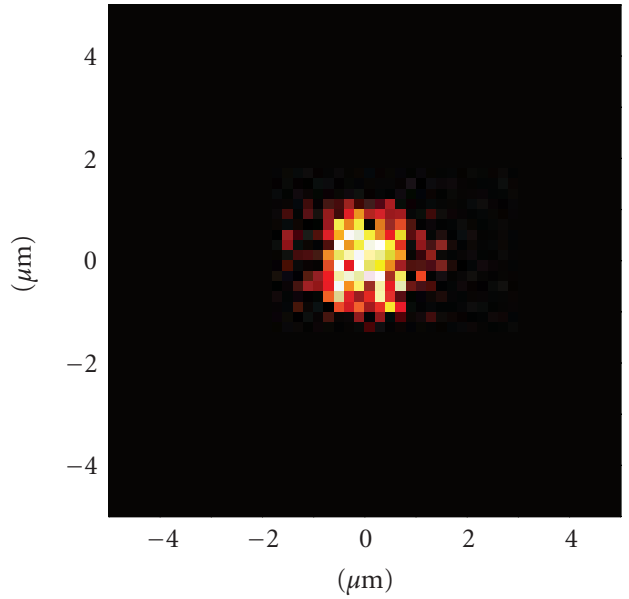

(b)

FIGURE 16: A small aperture double-reflection crossed pair system. (a) Traced rays; red: no reflection, green: 1 reflection, blue: 2 reflections, (b) Image of the focused spot.

TABLE 1: Summary of ray-tracing results. $G_{\text {geom }}$ is the gain calculated using the formula (22) or (25). $A_{\text {trace }}\left(\mathrm{mm}^{2}\right)$ and $G_{\text {trace }}$ are the geometric area and gain calculated from the ray-tracing. FWHM $(\mu \mathrm{m})$ is the width of the line-spread or point-spread function. $G_{\mathrm{Si}}$ is the gain calculated including the reflectivity of $1 \mathrm{keV}$ X-rays from a Silicon surface (uncoated). $G_{\mathrm{Au}}$ is the gain calculated including the reflectivity of $1 \mathrm{keV} \mathrm{X}$-rays from a Gold surface (channel walls coated).

\begin{tabular}{|c|c|c|c|c|c|c|}
\hline Configuration & $G_{\text {geom }}$ & $A_{\text {trace }}$ & FWHM & $G_{\text {trace }}$ & $G_{\mathrm{Si}}$ & $G_{\mathrm{Au}}$ \\
\hline Single Figure 12 & 58 & 3.5 & 16 & 58 & 9.0 & 23 \\
\hline Double Figure 13 & 100 & 11.1 & 30 & 70 & 8.2 & 16 \\
\hline Flat-curved Figure 14 & 56 & 2.5 & 9.0 & 40 & 0.06 & 6.9 \\
\hline Crossed Figure 15 & 6400 & 0.80 & 9.1 & 9660 & 326 & 1690 \\
\hline
\end{tabular}

focusing configuration is by far the best, despite the fact that the geometric aperture area is rather small. However, gain values of 100 s to 1000 s will only be achievable if the channel walls can be manufactured with low-surface roughness, $<2 \mathrm{~nm}$ rms, so that the TIS is kept low.

The results in Table 1 are for configurations in which the aperture ( $f$-ratio) was set to maximise the geometric collecting area. In these configurations, the width of the line-or point-spread functions is determined by the channel width $p$ which is why the FWHM values are $\sim 10 \mu \mathrm{m}$ or bigger. Instead of trying to maximize the aperture area we can choose to minimize the width of the focus while also providing a useful gain. At the same time we can look for configurations which are optimized for X-rays, including the X-ray reflectivity, rather than considering just the geometric response. We can reduce the focus size by shrinking the aperture, increasing the object and image distance and increasing the demagnification by making $s_{2} / s_{1}$ smaller. Figure 16 shows the ray-tracing results for a double-reflection crossed pair system with $s_{1}=s_{2}=$ $80 \mathrm{~mm}$, gap between the components $D=1 \mathrm{~mm}$, and aperture width $1 \mathrm{~mm}$. The simulation was run using X-ray reflectivities calculated for Silicon at $4.51 \mathrm{keV}$. The spot size and effective area are therefore representative of the idealised $\mathrm{X}$-ray performance at this energy. The radii of curvature were adjusted to give the minimum spot size FWHM =
$1.27 \mu \mathrm{m}$ and HEW $=1.28 \mu \mathrm{m} ; R_{1}=3180 \mathrm{~mm}$ and $R_{2}=$ $1077 \mathrm{~mm}$. The effective area was $A_{\text {eff }}=6.15 \times 10^{-4} \mathrm{~mm}^{-2}$. This is tiny because the grazing angles are very small, and most of the radiation goes straight through the channels without reflecting from the walls. A much larger area could be achieved if the channels were much narrower but such channels would be difficult to manufacture. Despite the low area, the gain is $G_{S i}=380$ which is comparable to the value obtained for the crossed system given in Table 1. Using a larger $f$-ratio, we can decrease the spot size, shift the response to higher $\mathrm{X}$-ray energies, and suffer a large decrease in effective area, but retain a high gain.

\section{Microprobing of Biological Cells}

A potential application of the MOAs described above is in the microprobing of biological cells. Research into radiation-induced cancers involves measuring the response of biological material—cells, subcellular components, and ultimately, tissue samples-to hard radiation. To do this, we need to focus $\mathrm{X}$-rays down to micrometre scale and to date this has been carried out using (primarily) carbon $\mathrm{K} \mathrm{X}$ rays (wavelength $44 \AA$, energy $284 \mathrm{eV}$ ) and zone plate optics $[17,18]$. These initial investigations are limited because carbon K X-rays are almost totally absorbed by single cells, so that tissue samples, of more relevance to living organisms, 
cannot be studied. For every $\sim 104$ cells killed by irradiation only one will mutate, so very many cells must be irradiated to track mutations, and in order to irradiate smaller cellular components, very small focal spot sizes are necessary. What is required is a focusing optic capable of delivering more flux per unit time (intensity) than a zone plate (which is limited by its aperture, typically a few hundred micrometres), while providing a focal spot of similar size. To facilitate tissue irradiation, higher X-ray energies are also needed but a zone plate focal length is proportional to energy, so a zone plate system is inherently achromatic and provides less demagnification of the source and hence larger spot sizes at higher energies. The fundamental equation which describes a zone plate is [19]

$$
r_{n}^{2}=n \lambda f+r_{0}^{2},
$$

where $\lambda$ is the wavelength of the X-rays being focused; $f$ is the focal length; $r_{0}$ is the radius of the first zone. The width of the $n$th zone can be calculated using

$$
d_{n} \approx \frac{\lambda f}{2 r_{n}}=\frac{r_{n}}{2 n} .
$$

In Section 5.2, we showed that an MOA system with a geometric aperture of $\approx 2 \mathrm{~mm}$, a channel aspect ratio (length: width) of $\approx 20: 1$, and a constant period of $20 \mu \mathrm{m}$ in $200 \mu \mathrm{m}$ thick silicon can produce small spot size and high gains provided that sidewall roughness is no more than a few nanometres. In particular, the double-reflection crossed system, Figure 16, was shown to provide a spot size of $\sim 1 \mu \mathrm{m}$ and gain of $\sim 380$ for an energy of $4.51 \mathrm{keV}$ (Ti $\mathrm{K}_{\alpha} \mathrm{X}$-rays). Ultimately, a double-reflection circular MOA configuration may be able to provide a performance better than this if the components can be manufactured and aligned.

For example, using the system illustrated in Figure 3 with $S=160 \mathrm{~mm}, D=1 \mathrm{~mm}, R=100 \mathrm{~mm}$, and $l=100 \mu \mathrm{m}$, gives $d \approx 10 \mu \mathrm{m}$ and $F=72.9 \mathrm{~mm}$, the same as the focal length of a zone plate (using (45) and (46)) of diameter $200 \mu \mathrm{m}$ and outer zone width $100 \mathrm{~nm}$ for Ti $\mathrm{K}_{\alpha} \mathrm{X}$-rays. For a point source of $\mathrm{Ti}_{\alpha} \mathrm{X}$-rays, the ray-tracing package Zemax [20] predicts an MOA focal spot diameter of $\approx 0.2 \mu \mathrm{m}$ while for a $5 \mu \mathrm{m}$ source, the focal spot diameter is $\approx 2 \mu \mathrm{m}$; the latter is dominated by the demagnified source size, and so it is the same for the zone plate and the MOA. With a smaller bending radius $R$ the value of $F$ for the MOA would be reduced, resulting in a smaller focal spot size. The only way to do this with a zone plate is to use a different optic with either a smaller diameter or a smaller outer zone width. With a sidewall roughness of $\approx 2 \mathrm{~nm}$, the MOA provides around two orders of magnitude more focused flux (higher gain) than the corresponding zone plate, primarily due to the larger aperture-the effective aperture diameter is $\approx 1 \mathrm{~mm}$ as towards the edge of the optic, the grazing angle is larger than the critical angle, so that the reflectivity decreases.

\section{Conclusion}

Active MOAs manufactured by etching Silicon have a great potential for the focusing of soft X-rays, $0.1-5 \mathrm{keV}$, into very small spot sizes. We have presented a comprehensive analysis of the various geometrical configurations that can be employed and simulation results which demonstrate this potential. The SXO consortium is currently working on refining the manufacturing process which produces the arrays of slots and optimising the way in which piezoelectric actuators can provide the necessary curvature and active adjustment of the focusing properties. Progress in these areas will be reported in subsequent publications.

\section{Acknowledgments}

The Smart X-Ray Optics consortium is funded by the UK Research Councils' Basic Technology programme, Grant code D04880X. The members of the consortium are University College London (including the Mullard Space Science Laboratory), King's College London (KCL), the Gray Cancer Institute (GCI), the University of Leicester, the Scottish Microelectronics Centre at the University of Edinburgh, the University of Birmingham, and STFC Daresbury Ltd. Silson Ltd. is an associate member. KCL has also received support from the European Science Foundation COST Action P7 "XRay and Neutron Optics", while KCL and GCI are supported by the COST Action MP0601 "Short Wavelength Laboratory Sources".

\section{References}

[1] P. D. Prewett and A. G. Michette, "MOXI: a novel microfabricated zoom lens for x-ray imaging," in Advances in X-Ray Optics, Proceedings of SPIE, pp. 180-187, August 2000.

[2] A. G. Michette, P. D. Prewett, A. K. Powell, S. J. Pfauntsch, K. D. Atkinson, and B. Boonliang, "Novel microstructured adaptive X-ray optics," Journal de Physique IV, vol. 104, pp. 277-280, 2003.

[3] M. Y. Al Aioubi, P. D. Prewett, S. E. Huq, V. Djakov, and A. G. Michette, "Design and fabrication of micro optical system for x-ray analysis of biological cells," in Opto-Ireland 2005: Optoelectronics, Photonic Devices, and Optical Networks, vol. 5825 of Proceedings of SPIE, pp. 640-646, Dublin, Ireland, 2005.

[4] M. Y. Al Aioubi, P. D. Prewett, S. E. Huq, V. Djakov, and A. G. Michette, "A novel MOEMS based adaptive optics for Xray focusing," Microelectronic Engineering, vol. 83, no. 4-9, pp. 1321-1325, 2006.

[5] July 2007, http://SmartXrayOptics.org.

[6] July 2007, http://www.rcuk.ac.uk/basictech/default.htm.

[7] A. Michette, T. Button, C. Dunare et al., "Active microstructured arrays for X-ray optics," in Advances in X-Ray/EUV Optics and Components II, vol. 6705 of Proceedings of SPIE, August 2007.

[8] A. G. Michette, S. J. Pfauntscha, S. Sahraeia et al., "Active microstructured X-ray optical arrays," in EUV and X-Ray Optics: Synergy between Laboratory and Space, vol. 7360 of Proceedings of SPIE, April 2009.

[9] C. Dunare, W. Parkes, T. Stevenson et al., "Microstructured optical arrays for smart x-ray optics," in EUV and X-Ray Optics: Synergy between Laboratory and Space, vol. 7360 of Proceedings of SPIE, April 2009. 
[10] D. R. Sanmartin, D. Zhang, T. Button et al., "Progress on the development of active micro-structured optical arrays for Xray optics," in Advances in X-Ray/EUV Optics and Components IV, vol. 7448 of Proceedings of SPIE, August 2009.

[11] C. A. MacDonald and W. M. Gibson, "Applications and advances in polycapillary optics," X-Ray Spectrometry, vol. 32, no. 3, pp. 258-268, 2003.

[12] G. J. Price, A. N. Brunton, G. W. Fraser et al., "Hard X-ray imaging with microchannel plate optics," Nuclear Instruments and Methods in Physics Research. Section A, vol. 490, no. 1-2, pp. 290-298, 2002.

[13] A. A. Ayn, X. Zhang, and R. Khanna, "Anisotropic silicon trenches 300-500m deep employing time multiplexed deep etching (TMDE)," Sensors and Actuators A, vol. 91, pp. 381385,2001

[14] H.-C. Liu, Y.-H. Lin, and W. Hsu, "Sidewall roughness control in advanced silicon etch process," Microsystem Technologies, vol. 10, no. 1, pp. 29-34, 2003.

[15] July 2007, http://www.comsol.com/.

[16] July 2007, http://www.star.le.ac.uk/ rw/q_v6/index.html.

[17] M. Folkard, K. M. Prise, C. Shao et al., "Understanding radiation damage to cells using microbeams," Acta Physica Polonica A, vol. 109, no. 3, pp. 257-264, 2006.

[18] A. G. Michette, "X-ray optics," in The Optics Encyclopedia, Th. G. Brown, K. Creath, H. Kogelnik, M. A. Kriss, J. Schmit, and M. J. Weber, Eds., pp. 3305-3371, John Wiley \& Sons, Berlin, Germany, 2003.

[19] G. R. Morrison, "Diffractive X-ray optics," in X-Ray Science and Technology, A. G. Michette and C. J. Buckley, Eds., p. 335, chapter 8, Institute of Physics Publishing, Bristol, UK, 1993.

[20] July 2007, http://www.zemax.com/. 